University of Wollongong

Research Online

Faculty of Engineering and Information

Faculty of Engineering and Information

Sciences - Papers: Part A

Sciences

$1-1-2013$

\title{
MIP-based stochastic security-constrained daily hydrothermal generation scheduling
}

J Aghaei

Shiraz University of Technology

M Karami

Iran University of Science and Technology

K M. Muttaqi

University of Wollongong, kashem@uow.edu.au

A Ahmadi

Iran University of Science and Technology Tehran

H A. Shayanfar

Iran University of Science and Technology Tehran

Follow this and additional works at: https://ro.uow.edu.au/eispapers

Part of the Engineering Commons, and the Science and Technology Studies Commons

Research Online is the open access institutional repository for the University of Wollongong. For further information contact the UOW Library: research-pubs@uow.edu.au 


\title{
MIP-based stochastic security-constrained daily hydrothermal generation scheduling
}

\begin{abstract}
This paper presents the application of a mixedinteger programming (MIP) approach for solving stochastic security-constrained daily hydrothermal generation scheduling (SCDHGS). Power system uncertainties including generating units and branch contingencies and load uncertainty are explicitly considered in the stochastic programming of SCDHGS. The roulette wheel mechanism and lattice Monte Carlo simulation (LMCS) are first employed for random scenario generation wherein the stochastic SCDHGS procedure is converted into its respective deterministic equivalents (scenarios). Then, the generating units are scheduled through MIP over the set of deterministic scenarios for the purpose of minimizing the cost of supplying energy and ancillary services over the optimization horizon ( $24 \mathrm{~h})$ while satisfying all the operating and network security constraints. To amore realistic modeling of the DHGS problem, in the proposed MIP formulation, the nonlinear valve loading effect, cost, and emission function are modeled in linear form, and prohibited operating zones (POZs) of thermal units are considered. Furthermore, a dynamic ramp rate of thermal units is used, and for the hydro plants, the multiperformance curve with spillage and time delay between reservoirs is considered. To assess the efficiency and powerful performance of the aforementioned method, a typical case study based on the standard IEEE-118 bus system is investigated, and the results are compared to each other in different test systems.
\end{abstract}

\section{Keywords}

security, constrained, daily, hydrothermal, generation, scheduling, mip, stochastic

\section{Disciplines}

Engineering | Science and Technology Studies

\section{Publication Details}

J. Aghaei, M. Karami, K. M. Muttaqi, H. A. Shayanfar \& A. Ahmadi, "MIP-based stochastic securityconstrained daily hydrothermal generation scheduling," IEEE Systems Journal, vol. 9, (2) pp. 615-628, 2015. 


\title{
MIP based Stochastic Security-Constrained Daily Hydrothermal Generation Scheduling
}

\author{
J. Aghaei, Member, IEEE, M. Karami, K.M. Muttaqi, Senior Member, IEEE, H. Shayanfar, and A. Ahmadi
}

\begin{abstract}
This paper presents the application of Mixed-Integer Programming (MIP) approach for solving the Stochastic Security-Constrained Daily Hydrothermal Generation Scheduling (SCDHGS). The power system uncertainties including generating units and branches contingencies and load uncertainty are explicitly considered in the stochastic programming of SCDHGS. The roulette wheel mechanism and Lattice Monte-Carlo Simulation (LMCS) are firstly employed for random scenario generation wherein the stochastic security constrained DHGS procedure is converted into its respective deterministic equivalents (scenarios). Then, the generating units are scheduled through a Mixed-Integer Programming (MIP) over the set of deterministic scenarios for the purpose of minimizing the cost of supplying energy and ancillary services over the optimization horizon (24 hours) while satisfying all the operating constraints and network security constraints. To more realistic modeling of DHGS problem, in the proposed MIP formulation, the nonlinear valve loading effect, cost and emission function are modeled in linear form and prohibited operating zones (POZs) of thermal units are considered. Furthermore, a dynamic ramp rate of thermal units is used and for the hydro plants, multi performance curve with spillage and time delay between reservoirs are considered. To assess the efficiency and powerful performance of mentioned method, a typical case study based on standard IEEE-118 bus system is investigated and the results are compared to each other in different test system.
\end{abstract}

Index Terms - Daily Hydrothermal Generation Scheduling (DHGS); Security-Constrained Unit Commitment (SCUC); Stochastic programming; Load uncertainty; Generator and branch outages

\section{Nomenclature}

Indices

$i, j$ : Indices of thermal and hydro unit

$\bar{i}, \bar{j}$ : Indices of bus

$t$ : index of time

$s$ : index of scenario

$u$ : index of unit in each bus

Constants

$\eta$ : Conversion factor equal to $3.6 \times 10^{-3}\left(\mathrm{Hm}^{3} \mathrm{~s} / \mathrm{m}^{3} \mathrm{~h}\right)$

$\Theta$ : Number of periods of the planning horizon

$L$ : Number of variable head

$M$ : Number of prohibited operation zones

J. Aghaei is with the Department of Electrical and Electronics Engineering, Shiraz University of Technology, Shiraz, Iran (Email: aghaei@sutech.ac.ir)

M. Karami and H. Shayanfar are with the Center of Excellence for Power System Automation and Operation, Department of Electrical Engineering, Iran University of Science and Technology, Tehran, Iran (Email:mahdi.karami@ee.iust.ac.ir, hashayanfar@,yahoo.com )

K.M. Muttaqi, is with APQRC, SECTE, University of Wollongong (Email kashem@uow.edu.au )

A. Ahmadi is with the Department of Electrical Engineering, Science and Research Branch, Islamic Azad University, Shiraz, Fars, Iran (Email: ahmadi.abdollah.janah@gmail.com )
NPL: Number of blocks of the piecewise linearization of the variable cost function

$N B$ : Number of system buses

$N U_{\bar{i}}:$ Number of units in $i^{\text {th }}$ bus

$N S$ : Number of scenarios in the scenario generation stage after scenario reduction

$N L$ : Number of load levels

$\pi_{s}$ : Probability of the $s^{\text {th }}$ scenario

$\pi_{s}^{\text {norm }}$ : Normalized probability of the $s^{\text {th }}$ scenario

$\underline{\theta}(j, t)$ : Minimum water discharge of unit $j$ at hour $t\left(\mathrm{~m}^{3} / \mathrm{s}\right)$

$\bar{\theta}(j, t)$ : Maximum water discharge of unit $j$ at hour $t\left(\mathrm{~m}^{3} / \mathrm{s}\right)$

$\tau_{i j}$ : Time delay between reservoir of plant $i$ and reservoir of plant $j$

(h)

$A_{i}$ : Shut down cost of unit $i(\$)$

$A_{j}$ : Start-up cost of unit $j(\$)$

$b_{n}(i)$ : Slope of block $n$ of fuel cost curve of unit $i(\$ / \mathrm{MWh})$

$b_{n}(j)$ : Slope of the volume block $n$ of the reservoir associated to unit $j$ $\left(\mathrm{m}^{3} / \mathrm{s} / \mathrm{Hm}^{3}\right)\left(1 \mathrm{Hm}^{3}=10^{6} \mathrm{~m}^{3}\right)$

$b_{n}^{k}(j)$ : Slope of the block $n$ of the performance curve of $\mathrm{k}$ unit $j$ $\left(\mathrm{MW} / \mathrm{m}^{3} / \mathrm{s}\right)$

$b e_{n}(i)$ : Slope of segment $n$ in emission curve of unit $i$

$D T(i)$ : Minimum down time of unit $i(\mathrm{~h})$

$e_{i}$ : Valve loading coefficient

$f_{i}$ : Valve loading coefficient

$F\left(p_{n-1}^{u}(i)\right)$ : Cost of generation of (n-1)th upper limit in fuel cost of unit $i$

$F(j, t)$ : Forecasted natural water inflow of the reservoir associated to plant $j$ in period $t\left(\mathrm{Hm}^{3} / \mathrm{h}\right)$

$K^{\lambda}(i)$ : Cost of the $\lambda^{\text {th }}$ discrete interval of the start-up cost of unit $i$ $(\$ / \mathrm{h})$

$I^{0}(i)$ : Initial status of unit $i(0 / 1)$

$M S R(i)$ : Maximum sustained ramp rate (MW/Min)

$M U$ : Maximum number of units that can be on at same time

$p(i), \bar{p}(i):$ Minimum/Maximum power output of unit $i(\mathrm{MW})$

$p_{n}(j)$ : Minimum power output of plant $j$ for performance curve $n(\mathrm{MW})$

$\bar{p}(j)$ : Capacity of plant $j$ (MW)

$p_{n}^{d}(i)$ : Lower limit of $n^{\text {th }}$ prohibited zone of unit $i$ (MW)

$p_{n-1}^{u}(i)$ : Upper limit of (n-1) th prohibited zone of unit $i(\mathrm{MW})$

$\underline{Q}(j)$ : Minimum water discharge of hydro plant $\mathrm{j}$ if is on $\left(\mathrm{m}^{3} / \mathrm{s}\right)$

$\bar{Q}_{n}(j)$ : Maximum water discharge of block $n$ of plant $j\left(\mathrm{Hm}^{3}\right)$

$R D L_{n}(i)$ : Ramp down limit for block $n(\mathrm{MW})$

$R U L_{n}(i)$ : Ramp up limit for block $n(\mathrm{MW})$

$s^{0}(i)$ : Time periods of unit $i$ has been shut-down at the beginning of the planning horizon $(\mathrm{h})$

$\bar{s}(j)$ : Maximum spillage of unit $j\left(\mathrm{~m}^{3}\right)$

$s_{\max }(i)$ : Maximum hour unit $i$ can be off (h)

$S D(i)$ : Shut-down ramp rate limit of unit $i(\mathrm{MW} / \mathrm{h})$

$S U(i)$ : Start-up ramp rate limit of unit $i(\mathrm{MW} / \mathrm{h})$

$U T(i)$ : Minimum up time of unit $i(\mathrm{~h})$ 
$U^{0}(i)$ : Time periods of unit $i$ has been on-line at the beginning of the planning horizon(h)

$v_{0}(j)$ : Minimum content of the reservoir associated to $\operatorname{plant} j\left(\mathrm{Hm}^{3}\right)$

$\mathrm{v}^{0}(\mathrm{j})$ : Reservoir content at the beginning of the study time $\left(\mathrm{Hm}^{3}\right)$

$\mathrm{v}^{\Theta}(j)$ : Reservoir content at the end of the study time $\left(\mathrm{Hm}^{3}\right)$

$v_{n}(j)$ : Maximum content of the reservoir $j$ associated to $n^{\text {th }}$ variable

head $\left(\mathrm{Hm}^{3}\right)$

Variables

$\pi_{t, s}$ : Probability of the $s^{\text {th }}$ scenario up to time $t$

$\gamma_{\mathrm{k}}$ : Probability of $k^{\text {th }}$ load level

$F O R_{\bar{i}, u}^{G}:$ Forced Outage Rate of $u^{\text {th }}$ unit in $i^{\text {th }}$ bus

FOR ${ }_{\bar{i}, \bar{j}}^{B}$ : Forced Outage Rate of branch between $i^{\text {th }}$ and $j^{\text {th }}$ buses

$w_{\bar{i}, u, s}^{G}$ : Status of the $u^{\text {th }}$ unit of $i^{\text {th }}$ bus in the $s^{\text {th }}$ scenario obtained

from MCS in the scenario generation stage (forced outage state or available)

$w_{\bar{i}, \bar{j}, s}^{B}$ : Status of branch between $i^{\text {th }}$ and $j^{\text {th }}$ buses in the $s^{\text {th }}$ scenario

obtained from MCS in the scenario generation stage (forced outage state or available)

$\delta_{n}(i, t)$ : Generation of block $n$ of fuel cost curve of unit $i$ at hour $t$

$\gamma(i, t)$ : Dummy variable (h)

$\psi_{n}(i, t)$ : Generation of block $n$ of unit $i$ at hour $t$ of valve point loadings curve

$\psi_{n}(j, t)$ : Volume block $\mathrm{n}$ for the reservoir of hydro plant $j$ at hour $t$ (MW)

$B(i, t)$ : Start-up cost of unit $i$ at hour $t(\$)$

$b_{n}(i)$ : Slope of power block $n$ of fuel cost curve of unit $i(\$ / \mathrm{MWh})$

$b_{n}^{l}(j)$ : Slope of the block $n$ of the performance curve 1 of hydro plant $j\left(\mathrm{MW} / \mathrm{m}^{3} / \mathrm{s}\right)$

$c(i, t)$ : Valve point loadings cost of unit $i$ at hour $t(\$)$

$F(i, t)$ : Fuel cost of unit $i$ at hour $t(\$)$

$p(i, t)$ : Real power generation of unit $i$ at hour $t$ (MW)

$p_{\min }(i, t, s), p_{\max }(i, t, s)$ : Lower and upper limit of real power generation

of unit $i$ at hour $t$ (MW)

$p(j, t)$ : Real power generation of unit $j$ at hour $t$ (MW)

$p(j, t, s), \bar{p}(j, t, s)$ : Lower and upper limit of real power generation of unit $\mathrm{j}$ at hour $t(\mathrm{MW})$

$Q(j, t)$ : Water discharge of unit $j$ at hour $t\left(\mathrm{~m}^{3} / \mathrm{s}\right)$

$q_{n}(j, t)$ : Water discharge of block $n$ of unit $j$ at hour $t\left(\mathrm{~m}^{3} / \mathrm{s}\right)$

$R D L(p(i, t))$ : Ramping down limit of unit $i$ at hour $t(\mathrm{MW})$

$R U L(p(i, t))$ : Ramping up limit of unit $i$ at hour $t$ (MW)

$s(i, t)$ : Time periods that unit $i$ has been shut-down at hour $t(\mathrm{~h})$

$s(j, t)$ : Spillage of the reservoir associated to unit $j$ at hour $t\left(\mathrm{~m}^{3} / \mathrm{s}\right)$

$v(j, t)$ : Water content of the reservoir associated to plant unit $j$ at hour $t$ $\left(\mathrm{Hm}^{3}\right)$

\section{Binary variables}

$w_{k, s}^{L}$ : Binary variable obtained from the roulette wheel mechanism in the scenario generation stage indicating whether $k^{\text {th }}$ load level in the $s^{\text {th }}$ scenario is occurred $\left(w_{k, s}^{L}=1\right)$ or $\operatorname{not}\left(w_{k, s}^{L}=0\right)$

$z_{i, u, s}$ : A binary variable indicating that the $u^{\text {th }}$ unit of $i^{\text {th }}$ bus in the $s^{\text {th }}$ scenario accepted or not in the energy market

$\beta_{n}(i, t, s): 1$ if block $n$ of fuel cost curve of unit $i$ at hour $t$ selected

$\beta_{n}(j, t, s): 1$ if variable head $n+1$ of unit $j$ at hour $t$ selected

$\chi_{n}(i, t, s): 1$ if power output of unit $i$ at hour $t$ has exceeded block $n$

$h_{n}(j, t, \mathrm{~s}): 1$ if the water discharge of unit $j$ at hour $t$ has exceeded block

$n$
$I(i, t, \mathrm{~s}): 1$ if thermal unit $i$ is on-line at hour $t$

$I(j, t, \mathrm{~s}): 1$ if hydro plant $j$ is on-line at hour $t$

$I d_{n}(i, t, \mathrm{~s}): 1$ if block $n$ of ramping down limit curve of unit $i$ at hour $t$ selected

$I u_{n}(i, t, \mathrm{~s}): 1$ if block $n$ of ramping up limit curve of unit $i$ at hour $t$ selected

${ }_{w}^{\lambda}{ }_{(i, t, s)}: 1$ if unit $i$ is started-up at the beginning of hour $t$ and it has been offline for $\lambda$ hours

$y(i, t, \mathrm{~s}): 1$ if unit $i$ is started-up at the beginning of hour $t$

$y(j, t, \mathrm{~s}): 1$ if unit $j$ is started-up at the beginning of hour $t$

$z(i, t, \mathrm{~s}): 1$ if unit $i$ is shut-down at the beginning of hour $t$

$z(j, t, \mathrm{~s}): 1$ if unit $j$ is shut-down at the beginning of hour $t$

Sets

G: Set of indices of the group units

I, J: Set of thermal units

$\mathrm{N}$ : Set of indices of the blocks of the piecewise linearization of the unit performance curve.

$\mathrm{T}$ : Set of indices of the periods of the market time horizon

S: Scenario numbers

SP: Stochastic parameters

$\Lambda$ : Set of the discrete intervals of the start-up cost function for thermal units

$\Omega_{\mathrm{j}}$ : Set of upstream reservoirs of plant $j$.

\section{Introduction}

Daily Hydrothermal Generation Scheduling (DHGS) determine the optimal usage of available hydro and thermal resources during a scheduling period of time (1 day-1week), in order to satisfy a forecasted energy demand at minimum total cost [1]. Therefore, the DHGS is a large-scale non-linear and complicated constrained power system optimization problem that can be solved using different optimization techniques as for example Lagrangian Relaxation (LR) [2], Dynamic Programming (DP) [3], Mixed Integer Programming (MIP) [4], Benders Decomposition (BD) [5] and various intelligent techniques [6-8]. A detailed literature review of optimization methods for solving DHGS problem is presented in [9].

The Independent System Operator (ISO) and GENeration COmpanies (GENCOs) are two main market participants with different goals. GENCOs try to maximize their profit and on the other side the ISO has the authority and responsibility to commit and dispatch system resources and curtail loads for maintaining the security constraints (i.e., balance load demands and satisfy fuel, environmental, and network security requirements) [10], [11]. Indeed, ISO determines an optimum schedule of generation units with the Standard Market Design (SMD) according to security-constrained unit commitment (SCUC) for minimizing the cost. Therefore, it will be important for ISO and GENCOs to schedule all units (thermal, hydro, wind and etc.) economically in a competitive environment [12], [13].

There are many works on the subject of the hydrothermal coordination (HTC) problem during the last years. In [14], DHGS is proposed and solved by a new Modified Adaptive Particle Swarm Optimization (MAPSO) technique. The impact of wind power plant on system operation cost is investigated in [15], while several constraints of units such as minimum up-time and down-time, minimum production, etc. are not considered in this paper. In [16] impacts of large-scale wind 
power on system operations from cost, reliability, and environmental perspectives with consideration of the units' limitation are studied. Also, it is assumed that no significant hydro power is installed in the system. But in this paper, HTC is investigated with practical constraints of units and system.

To obtain an optimal planning, it is very important for ISOs to consider rigorously and comprehensive model of the both hydro and thermal units in the DHGS. For this aim, in this paper the thermal and hydro subsystems are considered in planning of ISO against of [12-13],[17] and [18] that study thermal and hydro types separately without considering the network security constraints.

For more accuracy, more practical constraints of thermal and hydro units are taken into account. In [19-21], the valve loading effects cost is modeled in the form of a nonlinear sinusoidal function which is linearized in our framework. Inspired by [22], different dynamic ramp rate is also proposed in the proposed HTSS. Finally a general formulation is proposed for multi performance curve of hydro units based on [17]. Accordingly, the proposed HTSS includes a linear formulation for valve loading effects, fuel cost, emission function and fuel constraint, multi-performance powerdischarge curves of hydro units as well as units' minimum up/down time.

However, there are many uncertainties in the power system related to, e.g., electrical load variations and generator and branch outages. Thus, [23-24] have proposed a stochastic SCUC formulation for representing uncertainties in the availability of generation units and transmission lines, and inaccuracies in load forecasting. The component outages are simulated by the Monte Carlo Simulation (MCS).

In this study the Lattice Monte Carlo Simulation (LMCS) method and roulette wheel mechanism has been used for contingencies of generation units and transmission lines and load forecasting inaccuracies. To solve Stochastic SCDHGS problem, a two-stage solution method is proposed in this paper. In the first stage, the 24-h scenarios are generated using the roulette wheel mechanism and the LMCS. Besides, a scenario reduction technique is also presented in the paper to reduce the computational burden of the proposed UC procedure. In the second stage, the optimization problem of each generated scenario (selected by the scenario reduction technique) is solved by mixed-integer programming (MIP) method. Details of this two-stage solution method and how to implement and solve it are described in the following subsections.

The main objective of this paper is to minimize the total generation cost over the entire scenario tree by committing less expensive units while satisfying the corresponding constraints and dispatching the committed units economically.

As to the organization of the paper: Section 2 indicates proposed stochastic model for SCUC problem is formulated considering system's uncertainties. Section 3, the suggested model is applied to IEEE 118-bus system and the results are compared with deterministic method.

\section{Stochastic Model Description}

As mentioned before, ISO is in charge of maintaining fair, secure, and reliable operation of the power system. Power plant as well as transmission line failures may occur and forecasts of load and intermittent supply are inevitably uncertain. In overall, there are many uncertainties in the power system related to, e.g., electrical load variations and generator and branch outages. To cope with uncertainties, a sufficient resources reserve level must be considered in the system. Some methods are suggested for specification of reserve levels [25-27]. For simplicity, in the proposed method the volume of system reserve requirements are the constant that can be sold at hour $t$ and scenario $s$.

To solve the stochastic SCDHGS problem, a two-stage solution method is proposed in this paper. In the first stage, the 24-h scenarios are generated using the roulette wheel mechanism and the LMCS. Besides, a scenario reduction technique is also presented in the paper to reduce the computational burden of the proposed stochastic procedure. In the second stage, the optimization problem of each generated scenario (remained by the scenario reduction technique) is modeled and solved by MIP method. Details of this two-stage solution method are described in the following subsections.

\section{II.A First Stage: Scenario Generation and Reduction}

1) Scenario generation: Load uncertainty is assumed as the load forecast error. So, the probability distribution function of the system load forecast error can be obtained based on previous records of load. In this paper the total network load is considered as an independent variable to cope with the complication of problem when each load bus is an independent variable. So probability distribution function of each load bus can be determined according to its ratio of whole system load (load distribution factor). An example of the continuous distribution function of the system load forecast error along with its discretization is shown in Fig.1.

As can be seen in Fig.1 seven different interval are centered on the zero error mean (base state) so wide of each interval equal with the load forecast error standard deviation [28], [ 29]. The stochastic level of load to generate scenarios is modeled with roulette wheel mechanism [30, 31]. For this aim, at first, the probabilities of different load forecast levels are normalized such that their summation becomes equal to unity. Then the range of [0-1] is occupied by the normalized probabilities as shown in Fig.2. Naturally, whatever probability of load forecasting error level is more, will occupy more space of the roulette wheel. After that, random numbers are generated between 0 and 1 .

Each random number falls in one of the specified intervals related to the different load forecast error level in the roulette wheel. This means that load forecast level of related interval is selected for the respective scenario. Simultaneously with load uncertainty modeling, the unit/branch contingencies as the other source uncertainty are investigated by LMCS method based on FOR of them. Due to less difference of generated procedures by Lattice method than ordinary MCS method, in this paper this method is pursued to generate random numbers for scenarios. An n-point lattice rule of rank-r in d-dimension is describes as follows [24]:

$\sum_{l=1}^{r} \frac{k_{l}}{n_{l}}, v_{l} \bmod 1 \quad k_{l}=0,1, \ldots, n_{l}-1 \quad l=1, \ldots, r$ 
where $v_{1}, v_{2}, \ldots, v_{r}$ are randomly generated and linearly independent d-vector of integers. The number of random values needed to generate each scenario and variation of $k_{l}$ in rank $l(l=1, \ldots, r)$ is indicated by dimension $d$ and $n_{l}$ parameter respectively.

The convergence speed of LMCS method is greater than ordinary MCS and it can reach to same result with a smaller number of samples [24]. In Fig.3 are shown points generated by MCS and rank-1 lattice rule respectively. As can be seen, in LMCS method the distribution of points generated is more monotone than ones generated by MCS.

Therefore, LMCS based on the Forced Outage Rate (FOR) of generating units is implemented for the generating units' uncertainties. FOR of elements can be calculated as follow [32]:

$$
\operatorname{FOR}(n)=\frac{\lambda_{n}}{\lambda_{n}+\mu_{n}}
$$

where $\operatorname{FOR}(n)$ is FOR of $n^{\text {th }}$ unit, $\lambda_{n}$ and $\mu_{n}$ are failure rate and repair rate of $\mathrm{n}^{\text {th }}$ unit.

In this way, in each scenario, a random number between $[0,1]$ is generated for each generating unit and compared with it's FOR. If the generated number is greater than it's FOR, the unit is available and can partake in energy markets; otherwise, it is unavailable. $\mathrm{FOR}=5 \%$ means that the units is not available in $5 \%$ of time and will be available in $95 \%$ of time. Hence, if the generated number of unit is in $[0,0.05]$ margin, it will be not available and similarly if it falls in $[0.05,1]$ margin, it will be available. The procedure is used for all generators.

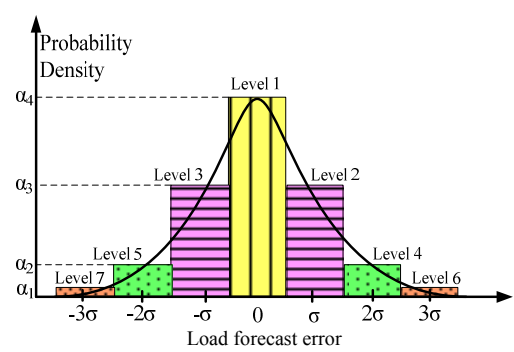

Fig.1: Typical discretization of the probability distribution of the load error

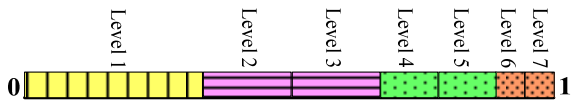

Fig. 2: Roulette wheel mechanism for the normalized probabilities of the load forecast levels
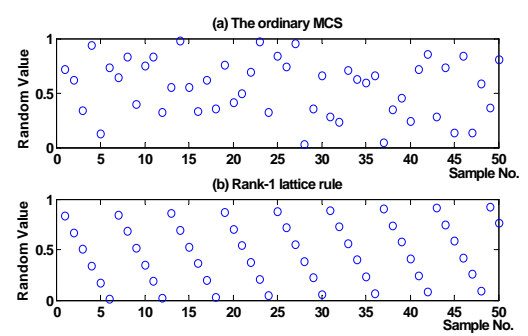

Fig. 3. Random points generated by

(a) the ordinary MCS and (b) rank-1 lattice rule
The determined load level by the roulette wheel mechanism plus the status of the generators and branches determined by the LMCS constructs one scenario of the stochastic optimization problem of SCDHGS for an hour. This procedure is repeated to generate the sightly number of scenarios for an hour.

2) Scenario reduction: Neutrally, as the number of generated scenarios becomes more, a wider range of optimization problem of the uncertainty space is covered and a better model of uncertainties will be obtained. But be careful that in this state the complexity of problem and cost of higher computation burden will be more. On the other hand generated scenarios with low probability increase time and calculations burden. For cope with this problem, the elimination of scenario with very low probability and scenarios that are very similar is implemented by scenario reduction techniques [33]. In this way, stochastic generated numbers for units/branches in one scenario may be different compared with another scenario, but both cause to a similar result that in this state both of them must be deleted. This reduction not only change method totality but also maintains a good approximation of the system uncertain behavior.

Scenario generation procedure explained in the previous section is implemented for $24-\mathrm{h}$ time period. Dispatching Center experiences show that when equipment (unit/branch) is obliged outage inadvertently, it will be remained out of grid to end of 24-h period. Scenario generation based on this method is known as an adaptive scenario generation algorithm. In this way, frist, $\mathrm{N}$ scenarios are randomly generated for the first hour (e.g., $\mathrm{N}=200$ ). Then NS scenarios most probable are selected with scenario reduction technique among generated scenarios (e.g., NS=20). Next, the selected scenarios are used for scenario generation process in the next hour, of course with considering of the intertemporal constraints mentioned prior. On the other hand, participation of the scenarios based on their probability in generating scenarios in the next hour give the better result. For this goal, it is necessary to calculate the each scenario probability from first 24 -h period to current hour. With these explanations, for next hour the number of generated scenarios from a specified selected scenario can be given as follow[34]:

$$
N_{t, s}=\text { Round }\left(\frac{\pi_{t-1, s}}{\sum_{s=1}^{N_{s}} \pi_{t-1, s}} \times N\right)
$$

where $N_{t, s}$ indicates number of scenarios in hour $\mathrm{t}$ generated from $s$ th scenario of hour $\mathrm{t}-1 . \pi_{t, s}$ indicates probability of $\mathrm{s}_{\mathrm{th}}$ scenario based on the information from hour 1 to hour $t$. Round operator is a function that rounds the number in brackets to the closest integer. As prior said, in the scenario generation for an hour, the outages of the previous hours should be considered. Here it is assumed that if a component trips in an hour, it is considered out of service for the remaining hours of that day. This means that after hour $\mathrm{t}-1$, the probability of the removed units will be equal 1 . Multiplying of generated $w_{\bar{i}_{, u}, t, s}$ and $w_{\bar{i}_{\bar{i},}, t, s}$ by MCS by $w_{\bar{i}, u, t-1, s}^{G}$ and $w_{\bar{i}, \bar{j}, t-1, s}^{B}$ respectively, guaranties above assumption. According 
above explanations and assumptions and value of the $w{ }_{\bar{i}, u, t, s}^{G}$ and $w \frac{B}{\bar{i}, \bar{j}, t, s}$ that represent state of units and branches respectively, the probability of $\mathrm{s}_{\mathrm{th}}$ scenario up to hour $\mathrm{t}$, i.e., $\pi_{t, s}$, can be computed as follows [34]:

$$
\begin{aligned}
\pi_{t, s}=\prod_{\tau=1}^{t}\left(\sum_{k=1}^{N L}\left(w_{k, \tau, s}^{L} \cdot \gamma_{k, \tau}\right) \cdot \prod_{i=1}^{N B} \prod_{u=1}^{N U_{i}} \eta_{\bar{i}, u, \tau, s}^{G} \cdot \prod_{i=1}^{N B} \prod_{j=1}^{N B} \eta_{\bar{i}, \bar{j}, \tau, s}^{B}\right) \\
\eta_{\bar{i}, u, \tau, s}^{G}=\left[\left(w_{\bar{i}_{, u, \tau},}^{G} \cdot\left(1-F O R_{\bar{i}, u}^{G}\right)+\left(1-w_{\bar{i}, u, \tau, s}^{G}\right) \cdot F O R_{\bar{i}, u}^{G}\right)\right] w_{\bar{i}, u, \tau-1, s}^{G} \\
+\left(1-w_{\bar{i}, u, \tau-1, s}^{G}\right) \\
\eta_{\bar{i}, \bar{j}, \tau, s}^{B}=\left[\left(w_{\bar{i}, \bar{j}, \tau, s}^{B} \cdot\left(1-F O R_{\bar{i}, \bar{j}}^{B}\right)+\left(1-w_{\bar{i}_{\bar{i}}, \bar{j}, \tau, s}^{B}\right) \cdot F O R_{\bar{i}, \bar{j}}^{B}\right)\right] w_{\bar{i}, \bar{j}, \tau-1, s}^{B} \\
+\left(1-w_{\bar{i}, \bar{j}, \tau-1, s}^{B}\right)
\end{aligned}
$$

where $\eta_{\bar{i}, u, t, s}^{G}$ and $\eta_{\bar{i}, \bar{j}, t, s}^{B}$ indicate share of $\mathrm{u}_{\mathrm{th}}$ unit of $\mathrm{i}_{\mathrm{th}}$ bus and branch between $i_{\text {th }}$ and $j_{\text {th }}$ buses in $\pi_{t, s}$, respectively. The given $\pi_{t, s}$ values from (4) are used in (3) to generating scenarios in adaptive scenario generation algorithm. It is noteworthy that the binary variables $w_{k, t, s}^{L}$ are determined by the roulette wheel mechanism. In fact, implementation of roulette wheel for a scenario in specified hour, load level $\mathrm{k}_{\mathrm{th}}$ is activated i.e. $w_{k, t, s}^{L}=1$ and the other load levels are deactivated so that $\sum_{k=1}^{N L} w_{k, t, s}^{L}=1$. Also the value of $w_{\bar{i}, u, t, s}^{G}$ and $w_{\bar{i}, \bar{j}, t, s}^{B}$ is appointed by MCS. This is important to note again that when a component of power system (unit or branch) is unavailable in hour $\tau$, it will remain unavailable in the next hours $(\tau \leq t)$ and the power system will be scheduled by the other remaining components. This means $w_{\bar{i}, u, \tau-1, s}^{G}=0$ or $\quad w_{\bar{i}, u, \tau-1, s}^{B}=0$ and consequently $\eta_{\bar{i}, u, \tau, s}^{G}=1$ and $\eta_{\bar{i}, u, \tau, s}^{B}=1$.

In implementation of the adaptive scenario generation algorithm for $24-\mathrm{h}$ period, it is noted that in hour $\tau, \mathrm{N}$ scenario are generated according to the NS probable selected scenario in hour $\tau-1$. Then the scenario reduction technique reduces them to the NS scenario. This procedure continue to hour 24, so in the end of adaptive algorithm the NS scenario 24-h will be achieved, so in each of them status of network equipments from view point of their availability or unavailability and also amount load for each hour is specified. The more participation opportunity for further likely scenarios in early hours is the most important characteristics of the adaptive scenario generation method. Also in this way eventuality of generating low value scenarios is weaker. We can capture more the uncertainty spectrum compared with the uniform scenario generation procedure. After generating of scenarios to compute their contingency is sufficient to put 24 instead $t$ in equation (7).

$$
\pi_{s}=\pi_{24, s}
$$

As can be seen in this stage the status of the units, branches and loads is defined by the stochastic methods. Really at the end of this stage load level and the units which can be available and participate in the UC are determined, but it not guaranty all units committed in the second stage. In the next subsection the formulation of optimization problem of generated scenarios to determining situation of units according to the network constraints and operating characteristics is represented.

\section{II.B Second Stage: Stochastic Security Constrained Daily Hydrothermal Generation Scheduling}

In the following, the objective function and its different parts of SCDHGS will be explained clearly.

\section{A. Objective function}

Our proposed objective function is to determine the optimal usage of available hydro and thermal resources during a scheduling period of time ( 1 day), in order to satisfy a forecasted energy demand at minimum total cost. This objective function is formulated as follows:

$\operatorname{Cost}^{\text {total }}=\sum_{s} \pi^{\text {norm }}(s) \cdot \operatorname{Cost}(s)$

$\operatorname{Cost}(s)=\sum_{t \in T}\left\{\begin{array}{l}\sum_{i \in I}\left[F(i, t, s)+A_{i} Z(i, t, s)+B(i, t, s)+C(i, t, s)\right] \\ +\sum_{j \in J} A_{j} y(j, t, s)\end{array}\right\}$

where the first term represents thermal operating cost including fuel, shutdown, startup costs and valve point loadings cost, the second term represents the startup cost of hydro units over the given period.

The start-up costs of hydro units are considered in the model to prevent unnecessary commitments, the loss of water during start-up period, wear and tear of the windings and mechanical equipment, and malfunctions in the control equipment [17].

\section{B. Network constraints}

\section{B.1 System power balance}

When minimizing the total cost in power systems, the total generation of hydro and thermal plants should be equal to the total system demand plus the transmission network loss. But, for simplicity the network loss is not considered in this paper. This gives the equality constraint [28].

$\sum_{i \in I} p(i, t, s) * I_{i t s}+\sum_{j \in J} p(j, t, s) * I_{j t s}=\mathrm{P}_{\mathrm{D}, \mathrm{t}} \quad \forall \mathrm{t} \in \mathrm{T}$

\section{B.2 System total reserve requirements}

ISO manages an operating reserve (OR), which is essential to maintaining the reliability of electricity system by ensuring that there is always enough supply to meet the demand for electricity. OR is stand-by capacity that is kept online in case the power system suffers a severe strain and reserve power is required. Addition to the OR an enough Spinning Reserve (SR) capacity should be considered in system planning. This matter can be described as follow [35]:

$$
\begin{aligned}
& \sum_{i=1}^{N G} R_{S, i t s} * I_{i t s} \geq R_{S, t s} \\
& \sum_{i=1}^{N G} R_{O, i t s} * I_{i t s} \geq R_{O, t s} \quad \forall t=1, \ldots, N T \quad \forall \mathrm{s} \in \mathrm{S}
\end{aligned}
$$

\section{B.3 Network security constraints}

The security constraints can be obtained based on DC power flow or linear power flow model. In this study the DC power flow model are considered in the program due to its more 
precision. In this model the well-known Kirchoff's current law (KCL) and Kirchoff's voltage law (KVL) are implemented to control of the physical flow in the transmission grid. This is done while the just KCL is used in linear power flow model. The DC power flow model's formulations are described as below [36]:

$$
\begin{array}{cc}
\left(\sum_{i=1}^{N G_{b}}\left(P_{i t s} * I_{i t s}+P_{j t s} * I_{j t s}\right)-P_{b t}^{D}=\sum_{l=1}^{L_{b}} P_{l t}\right. \\
F_{l t}=\frac{1}{X_{l}}\left(\delta_{l s}-\delta_{l r}\right) & \forall b, \forall t \in T
\end{array}
$$

Transmission flow limits in the base case:

$$
-F_{l t}^{\max } \leq F_{l t} \leq F_{l t}^{\max } \quad \forall l, \forall t \in T
$$

\section{Hydro units' model}

In this section the constraints of hydro units are taken. The generated power of hydro unit is a nonlinear function of water discharge and variable head of the associated reservoir which has been shown in Fig.4. For more accuracy multi performance curves must be used because this concept is very important when storage capacity of reservoirs is small and generated power depends on hydro unit head. So, in this study multi-performance curves of hydro plants are considered in problem according to the related head of reservoirs. It is assumed that the hydro units have L performance curves. In this way, the head dependent reservoirs are modeled with MIP formulations as hydro units which are connected in parallel and series (Fig.5).

\section{C.1 Linear formulations for volume and multi performance curves}

The linear formulations of hydro power units with L performance curves are as the following equations:

$$
\begin{aligned}
v(j, t, s) \geq & v_{0}(j) ; \forall j \in J \\
v(j, t, s) \leq & v_{L}(j) \beta_{L-1}(j, t, s)+\sum_{n=2}^{L} v_{n-1}(j)\left[\beta_{n-2}(j, t, s)-\beta_{n-1}(j, t, s)\right] \\
v(j, t, s) \geq & v_{L-1}(j) \beta_{L-1}(j, t, s) \\
& \quad+\sum_{n=3}^{L} v_{n-2}(j)\left[\beta_{n-2}(j, t, s)-\beta_{n-1}(j, t, s)\right] \\
\beta_{1}(j, t, s) \geq & \beta_{2}(j, t, s) \geq \ldots . . \beta_{L-1}(j, t, s)
\end{aligned}
$$

Equations (15)-(18) specify the performance curves according to the water value. Eq.(15) represents the volume of hydro plant must be bigger than its minimum limit. The equations (16) and (17) choose the right curve for head according to the content level. Eq.(18) is used to prevent from combination of 0-1 binary variables $\beta_{\mathrm{n}}(\mathrm{j}, \mathrm{t}, \mathrm{s})$.

\section{C.2 Piecewise linearization of variable head power-discharge performance curves}

As mentioned earlier the generated power by hydro plant is a nonlinear function of several factors as turbine discharge rate and the net head or, equivalently, the volume of the stored water in the reservoir. Because of this reason, in this paper is used from multi performance curve as shown in Fig.4. Also for simplification in calculations, a linear formulation between hydro power and discharged water corresponding performance

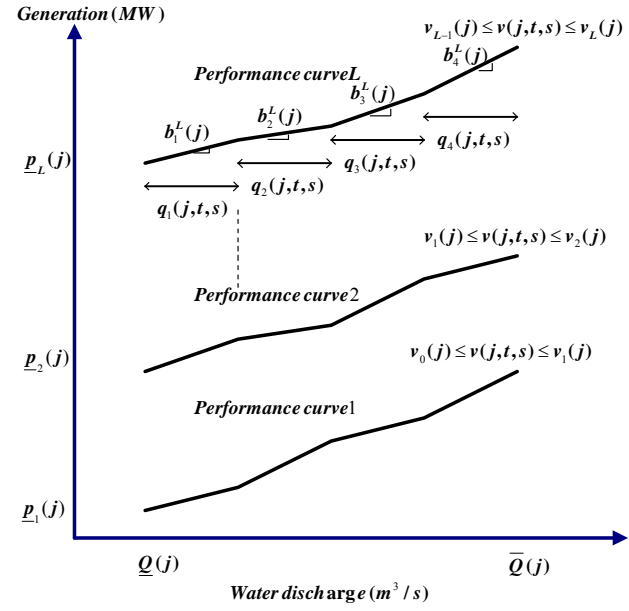

Fig.4. Three-dimensional piecewise linear non-concave unit performance curve for hydro plant $\mathrm{j}$ at hour $\mathrm{t}$

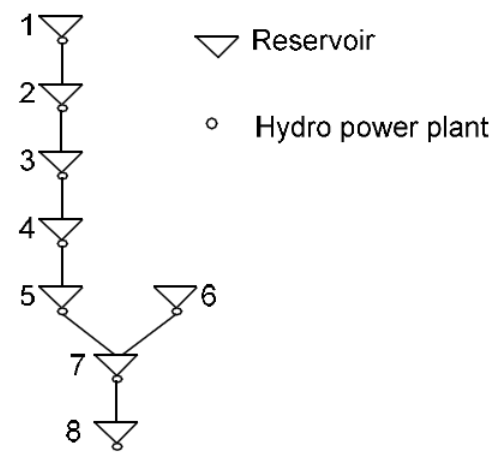

Fig.5. Hydraulic topology of the river basin

curve is used in this study as follows:

$$
\begin{aligned}
& p(j, t, s)-\underline{p}_{k}(j) I(j, t, s)-\sum_{n \in N} q_{n}(j, t, s) b_{n}^{k}(j) \\
& -\bar{p}(j)\left[(k-1)-\sum_{n=1}^{k-1} \beta_{n}(j, t, s)+\sum_{n=k}^{L-1} \beta_{n}(j, t, s)\right] \leq 0 \quad, 1 \leq k \leq L \\
& p(j, t, s)-\underline{p}_{k}(j) I(j, t, s)-\sum_{n \in N} q_{n}(j, t, s) b_{n}^{k}(j) \\
& +\bar{p}(j)\left[(k-1)-\sum_{n=1}^{k-1} \beta_{n}(j, t, s)+\sum_{n=k}^{L-1} \beta_{n}(j, t, s)\right] \geq 0,1 \leq k \leq L
\end{aligned}
$$

where $p(j, t, s)$ is the generated power by the hydro unit $j$ at hour $\mathrm{t}, \mathrm{P}_{\mathrm{k}}(\mathrm{j})$ is the minimum generated power of the head $\mathrm{k}$ which is determined by $\beta_{\mathrm{n}}(\mathrm{j}, \mathrm{t})$. Also, $\mathrm{P}(\mathrm{j})$ is the capacity of hydro unit $j$, and $q_{n}(j, t, s)$ is the water discharge of the block $n$. Finally, $b_{n}{ }^{k}(j)$ is the slope of the block $n$ of the variable head $k$ of hydro unit $\mathrm{j}$.

\section{C.3 Water discharge limits}

Water discharge of hydro plant $j$ is as the following equations: $Q(j, t, s)=\underline{Q}_{k}(j) I(j, t, s)-\sum_{n \in N} q_{n}(j, t, s) \quad \forall \mathrm{j} \in \mathrm{J}, \forall \mathrm{t} \in \mathrm{T}$

Also, for flooding prevention and irrigation requirements the following constraint is needed.

$\underline{Q}(j, t, s) \leq Q(j, t, s)+s(j, t, s) \leq \bar{Q}(j, t, s) \quad \forall j \in J, \forall t \in T$

where, $s(j, t, s)$ is the spillage of hydro plant $j$ at hour $t$. Here, we use two blocks for linearization of the spillage-volume curve [8], which can be incorporated into the MIP problem. 


$$
\begin{array}{ll}
q(j, t, s) \leq \overline{Q_{1}}(j) I(j, t, s) & \forall \mathrm{j} \in \mathrm{J}, \forall \mathrm{t} \in \mathrm{T}, \forall \mathrm{s} \in \mathrm{S} \\
q(j, t, s) \geq \overline{Q_{1}}(j) h_{1}(j, t, s) & \forall \mathrm{j} \in \mathrm{J}, \forall \mathrm{t} \in \mathrm{T}, \forall \mathrm{s} \in \mathrm{S} \\
q(j, t, s) \leq \overline{Q_{n}}(j) h_{n-1}(j, t, s) & \forall \mathrm{j} \in \mathrm{J}, \forall \mathrm{t} \in \mathrm{T}, \forall \mathrm{s} \in \mathrm{S} \\
q(j, t, s) \geq \overline{Q_{n}}(j) h_{n}(j, t, s) & \forall \mathrm{j} \in \mathrm{J}, \forall \mathrm{t} \in \mathrm{T}, \forall \mathrm{s} \in \mathrm{S}
\end{array}
$$

\section{C.4 The other constraints of hydro units}

Addition to the mentioned constraints, the other constraints of hydro units such as initial and final volume [17], Water balance [37] and operating services [38] are considered in the problem. Details of these constraints are given in mentioned references.

\section{Thermal units' model}

This subsection deal with the linearization of all nonlinear equations related to thermal units.

\section{D.1 Linear fuel cost function considering POZ}

The fuel cost function of the thermal units is represented by a quadratic function in many studies. But in practical operation of the power system, several thermal units have some POZs that the units should not operate in those. This limitation refers to the steam valve operation or vibration in its shaft bearing and some faults in the machines or their accessories such as pumps or boilers, etc. Considering the $\mathrm{POZ}$ causes to have the discontinues curves. In this study the quadratic cost function of the thermal units are approximated by a set of piecewise blocks as shown in Fig. 6 .

\section{D2. Valve point loadings cost}

In the thermal units with multi-valve steam turbines ,when steam admission valves are first opened, a sudden increase in losses is registered which results in ripples in the cost function (Fig.7). This effect is known as a valve point loading. To considering valve-point effects, sinusoidal functions are added to the quadratic cost functions as follows [39]:

$$
F_{i}\left(P_{i}\right)=a_{i}+b_{i} P_{i}+c_{i} P^{2}+a b s\left(e_{i} \sin \left(f_{i}\left(P_{i \text { min }}-P_{i}\right)\right)\right) \quad \forall i \in I
$$

where $e_{i}$ and $f_{i}$ are the coefficients of $i_{\text {th }}$ generator.

As can be seen, when sinus term is added to the cost function will cause the problem to be non-convex and nonlinear. For this reason in proposed MIP model, a linear model is considered instead (27) as cost function of thermal units as follows (Fig.8.)

$$
\begin{aligned}
& C(i, t, s)=\frac{2 e_{i} f_{i}}{\pi}\left\{\begin{array}{l}
\sqrt{2} \sum_{n=0}^{k_{i}}\left[\psi_{4 n+1}(i, t, s)-\psi_{4 n+4}(i, t, s)\right] \\
+(2-\sqrt{2}) \sum_{n=0}^{k_{i}}\left[\psi_{4 n+2}(i, t, s)-\psi_{4 n+3}(i, t, s)\right]
\end{array}\right\} \\
& p(i, t, s)=\underline{p}(i) I(i, t, s)+\sum_{n=0}^{k_{i}}\left[\begin{array}{l}
\psi_{4 n+1}(i, t, s)+\psi_{4 n+2}(i, t, s) \\
+\psi_{4 n+3}(i, t, s)+\psi_{4 n+4}(i, t, s)
\end{array}\right] \\
& \frac{\pi}{4 f_{i}} \chi_{1}(i, t, s) \leq \psi_{1}(i, t, s) \leq \frac{\pi}{4 f_{i}} I(i, t, s) \forall i \in I, \forall t \in T, \forall s \in S \\
& \frac{\pi}{4 f_{i}} \chi_{n}(i, t, s) \leq \psi_{n}(i, t, s) \leq \frac{\pi}{4 f_{i}} \chi_{n-1}(i, t, s) ; \\
& \forall i \in I, \forall t \in T, n=2,3, \ldots, x_{i}, \forall s \in S
\end{aligned}
$$

$\chi_{n}(i, t, s) \in\{0,1\} \quad \forall i \in I, \forall t \in T, \forall s \in S, n=1,2, \ldots, x_{i}$

where, $\psi_{\mathrm{n}}(\mathrm{i}, \mathrm{t})$ is power generated by $n_{\text {th }}$ block and $k_{i}=$ floor $\left[f_{i} \frac{p_{\max }(i)-p_{\min }(i)}{\pi}\right]$ and $x_{i}=$ floor $\left[4 f_{i} \frac{p_{\max }(i)-p_{\min }(i)}{\pi}\right]$.

Eq.(29) determine the generating power of unit $i$ at hour $t$ as if it is ON, the power output will be sum of the minimum power output, plus the power generated in each block. According to Eq.(30), the generated power in the first block should be in specified margin, greater than zero and smaller than or equal to $\pi / 4 f i$ that is "power length" of each block. If a unit be OFF, $I(i, t)$ will be zero and consequently its output power oblige to be zero. In Eq. (31) to (32) the binary variable $\chi_{n}(i, t)$ are used to limit the generated power in each block so it will be 1 if the generated power of unit $i$ at hour $t$ has exceeded block $n$.

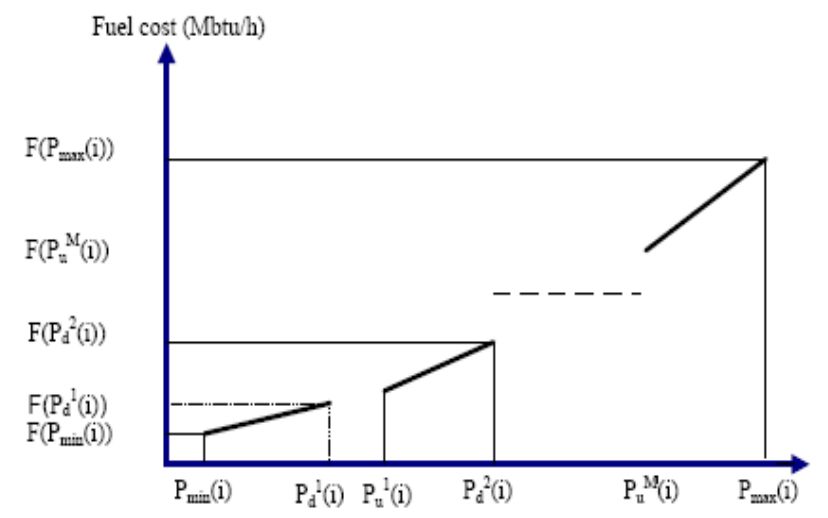

Fig.6. Piecewise linear fuel cost curve with $\mathrm{M}$ prohibited operating zones

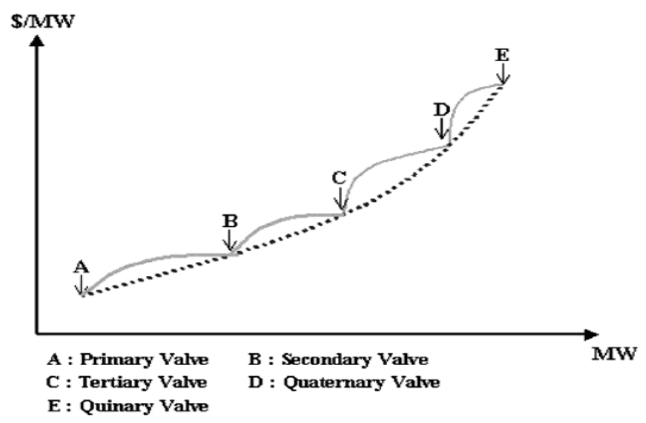

Fig.7. Example of cost function with 5 valves.

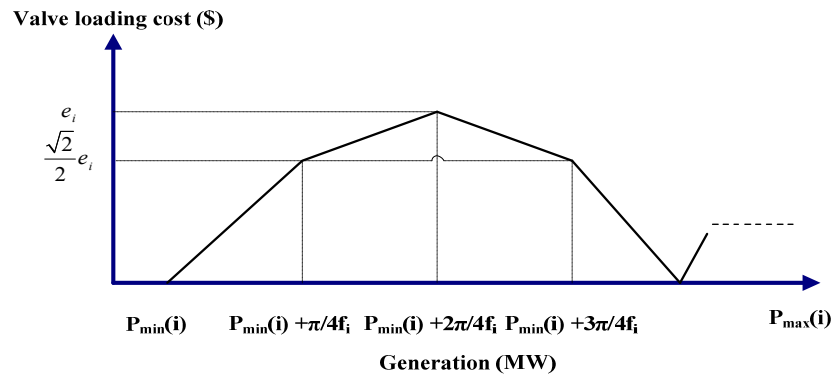

Fig. 8. Linear approximation of absolute sinus function of valve loading cost

D3.Dynamic Ramping Up/Down Limit

Inspired by [22], the proposed dynamic ramp rate is a function of thermal units is:

$R D L(p(i, t, s))=\sum_{n=1}^{M+1} R D L_{n}(i) \beta_{n}(i, t, s)$ 
$R U L(p(i, t, s))=\sum_{n=1}^{M+1} R U L_{n}(i) \beta_{n}(i, t, s)$

According to Eq.(22) and (23), dynamic ramp rate is related to thermal units by $\beta_{n}(i, t, s)$. Detailed formulations of dynamic ramp rate are presented in [22].

\section{D4. Generation thermal unit capacity limits}

The upper and lower limit constraints of thermal units including the ramp up limit (RUL) and ramp down limit (RDL) can be written as:

$$
\begin{aligned}
& \underline{p}(i) I(i, t, s) \leq p(i, t, s) \leq \bar{p}(i, t, s) \\
& \bar{p}(i, t, s) \leq \bar{p}(i)\{I(i, t, s)-z(i, t+1, s)\}+S D(i) Z(i, t+1, s) \\
& p(i, t-1, s)-p(i, t, s) \leq S D(i) Z(i, t, s)+R D L(p(i, t, s)) \\
& p(i, t+1, s)-p(i, t, s) \leq S U(i) y(i, t+1, s)+R U L(p(i, t, s))
\end{aligned}
$$

\section{D5. The other constrains of thermal units}

In order to sustain sudden events of power systems such as transmission lines or generators outages, operating services (spinning reserve and non-spinning reserve) are considered [40]. The other constraints of are [12, 37]: time varying startup cost function, Minimum Up-Time (MUT) and Minimum Down-Time (MDT), and logical status of commitment. The fuel limit constraints are taken from [24, 40, 41].

\section{II.C Third Stage: Scenario Aggregation}

The idea of stochastic security-constrained HTC is to construct or sample possible options for uncertain circumstances, solve the deterministic SCDHGS problem for the possible options, and select a good combination of the outcomes to represent the stochastic solution. Two methods are usually considered for scenario aggregation of the stochastic SCDHGS [32, 42]. In this paper the weightedaverage (expected value) method is used for aggregation of different scenarios result paper to determine total daily operating cost of problem. In this way, the solutions obtained from different scenarios are aggregated based on the probability laws to yield a single solution, describing the most probable outcome of the power system based on the evaluated scenarios, considered as the result of the proposed stochastic SCDHGS framework. As stated, the LMCS method is implemented to simulate the random characteristics of power systems load and then the scenario aggregation technology is used to convert the stochastic variables of the stochastic SCDHGS problem into deterministic ones. A major advantage of scenario aggregation technique is that not only individual scenarios become simple to interpret but also the underlying problem structure is preserved. After running the proposed SCDHGS scheme for the accepted scenarios resulted from the scenario reduction, the results are aggregated according to the probability of scenarios to get the expected results of the formulation of hydrothermal scheduling considering uncertainties.

The aggregation is done for the scenario dependent decision variables $I(i, t, \mathrm{~s}), I(j, t, \mathrm{~s}), F(i, t, \mathrm{~s}), p(i, t, s), p(j, t, s), R(i, t, s), R(j, t, s)$ of the optimization problem. The aggregation is done as:

$$
f=\sum_{s=1}^{N S} \pi_{s}^{\text {nom }} f_{s} \quad \forall s \in S
$$

where, $f$ is the variable that is aggregated and $f_{s}$ is the variable value at scenario $\mathrm{s}$. It is noted that the objective function of the proposed formulation for SCDHGS problem in equation (8) is also an aggregation of the objective function values of the scenarios.

\section{Numerical Results}

A modified IEEE 118-bus test system in Fig. 9 is used to test the proposed algorithm for the stochastic security constrained day-ahead hydrothermal power scheduling [41]. This system contains 54 thermal units which are 10 oil-fired, 11 gas-fired and 33 coal-fired units. To model hydro units, eight hydro units are considered and their required data are taken from [8]. The POZ data and valve loading coefficients and also base deterministic market loads for energy and reserve (spinning and non-spinning) are taken from [43]. Based on [30], the start-up cost for thermal units is linearized in 10 blocks. Also, based on [44] the emission function of $\mathrm{SO}_{2}$ and $\mathrm{NO}_{\mathrm{x}}$ becomes linear in 4 blocks. It is assumed that both of $S U(i)$ and $S D(i)$ are equal to $0.7 p_{\max }(i)$; the generated $\mathrm{SO}_{2}$ and $\mathrm{NO}_{\mathrm{x}}$ of thermal units are $1000 \mathrm{lbs}$ (in the period of starting-up and shuttingdown period). Due to lack of information data, without the loss of generality, fix ramp rate data is used. For hydro units, 3 performance curves is used that each of them is linearized in 4 blocks as shown in Fig. 4. Total spinning and non-spinning reserve can be sold at each hour and scenario is $500 \mathrm{MW}$. Other data for thermal units are taken from [43].

The paper aims at the development of MIP models for the cost effective scheduling of electric power in a hydro thermal generation system under system uncertainties such as forced outages of system components and load forecasting inaccuracies which considers more practical constraints and rigorous modeling of thermal and hydro units than previous works in the area to the best of our knowledge.

The practical constraints of thermal and hydro generation units that detailed their modeling are mentioned in Section 2 and additional system-wide constraints such as fuel constraints and emission limits $[24,39,45]$ and spinning and operating reserve [46] requirements are considered in the stochastic optimization framework. Thermal units $5,10,11,28,36,43$, 44 and 45 have valve loading effect cost and thermal units 7 , $10,30,34,35$ and 47 have POZs limitations. The proposed MIP optimization model of stochastic security constrained DHGS is implemented on a Pentium IV, $3 \mathrm{GHz}$ with $1 \mathrm{~GB}$ of RAM using MILP solver CPLEX 9.0 in the GAMS environment [47].

With the use of roulette wheel mechanism for modeling the uncertainty of load, 200 scenarios, including daily load profiles, are generated. It imposes a high computational burden to solve the stochastic SCDHGS problem for all of these scenarios. So, the set of generated scenarios (200 daily load profiles) is reduced using the scenario reduction technique. The generated similar scenarios and scenarios with probability lower than 0.003 are discarded. Number of remaining scenarios after scenario reduction is equal to 20 , which results in $200 / 20=10$ filtering ratio. So, the scenario reduction technique significantly reduces the computation burden of the proposed stochastic SCDHGS framework. At 
the same time, the most probable and dissimilar scenarios are retained while maintaining a good approximation of the uncertain behavior of these uncertainty resources. For the remaining set of scenarios, the proposed stochastic MILP model of SCDHGS is run considering the status of generators and branches in each individual scenario. Selected scenarios, their normalized probability and total daily operating cost are presented in Table I.

\section{Case1: Stochastic security constrained hydrothermal coordination}

In the state that security constraints are considered (inequalities 12, 13, and 14), the minimum and maximum daily operating cost are related to scenario 15 and scenario 8 with $769924.1 \$$ and 897413 \$ respectively. The commitment schedules for these scenarios are shown in Tables II- III that 1 and 0 represent $\mathrm{ON} / \mathrm{OFF}$ states of units at different hours, and hour 0 represents the initial condition. According to simulation of scenario 15 and 8 , the following results are obtained:

In scenario 15, the thermal units 1,2 and 3 which have the high production cost is remained off on all period of operation. While in the scenario 8 , these three units at the end of the day hours (from hour 15, 16 or 19 to 24) have been forced to turn on and be connected to the grid. In the scenario 15, the thermal plant 16 is not committed in all of 24 hours, while in the scenario 8, this plant is committed at hour 1 to 22; something like this state is seen for unit 53. Also, in the scenario 15 , the thermal plants 21 and 24 are $\mathrm{ON}$ at total of 24 hours while in scenario 8 , these are $\mathrm{ON}$ only at hour 9 to 24 . The expensive unit of plant 52 is committed at hour 1 to 13 in scenario 15 while it will be OFF at total of 24 hours in scenario 8. Also the inexpensive plant 4 has been ON more hours in scenario 15 than scenario 8 .

\begin{tabular}{|c|c|c|c|c|}
\hline \multirow{2}{*}{ No. } & \multirow{2}{*}{$\begin{array}{c}\text { Scenario } \\
\text { No. }\end{array}$} & \multirow{2}{*}{$\begin{array}{l}\text { Normalized } \\
\text { Probability }\end{array}$} & \multicolumn{2}{|c|}{ Daily operating cost(\$) } \\
\hline & & & With Security & Without security \\
\hline 1 & 1 & 0.475 & 782067 & 726719 \\
\hline 2 & 26 & 0.022 & 864117 & 819447 \\
\hline 3 & 28 & 0.085 & 827608 & 773933 \\
\hline 4 & 39 & 0.018 & 775200 & 717774 \\
\hline 5 & 40 & 0.063 & 880708 & 816517 \\
\hline 6 & 45 & 0.018 & 792711 & 731755 \\
\hline 7 & 52 & 0.028 & 868775 & 804123 \\
\hline 8 & 56 & 0.026 & 897413 & 787427 \\
\hline 9 & 78 & 0.021 & 828908 & 774777 \\
\hline 10 & 79 & 0.023 & 770930 & 732146 \\
\hline 11 & 82 & 0.022 & 882141 & 838416 \\
\hline 12 & 87 & 0.021 & 789907 & 730819 \\
\hline 13 & 97 & 0.017 & 809952 & 752936 \\
\hline 14 & 101 & 0.017 & 880296 & 827296 \\
\hline 15 & 105 & 0.022 & 769924 & 723662 \\
\hline 16 & 133 & 0.017 & 803521 & 722218 \\
\hline 17 & 145 & 0.022 & 856735 & 759420 \\
\hline 18 & 158 & 0.031 & 833899 & 780606 \\
\hline 19 & 164 & 0.035 & 791931 & 726773 \\
\hline 20 & 194 & 0.020 & 879415 & 822611 \\
\hline
\end{tabular}

So, inexpensive plant 4 is connected to the grid at total of 24 hours while in the scenario 8 it has been committed only at hour 9 to 24 . The hydro units 2, 5, 6 and 7 in scenario 15 are committed more hours than scenario 8 .

The total output power of hydro and thermal plants in scenarios 15 are $14779.11 \mathrm{MW}$ and $57134.94 \mathrm{MW}$ and the power in the scenario 8 are 15139.99 MW and 59662.01 MW, respectively. As can be seen, in scenario with lower production cost, i.e. the scenario 15 , the amount of hydro plants production is little more. Also, the ratio of the hydro generated power to the total generated power with hydro and thermal plants is $20.55 \%$ and $20.24 \%$ in the scenarios 15 and 8 , respectively.

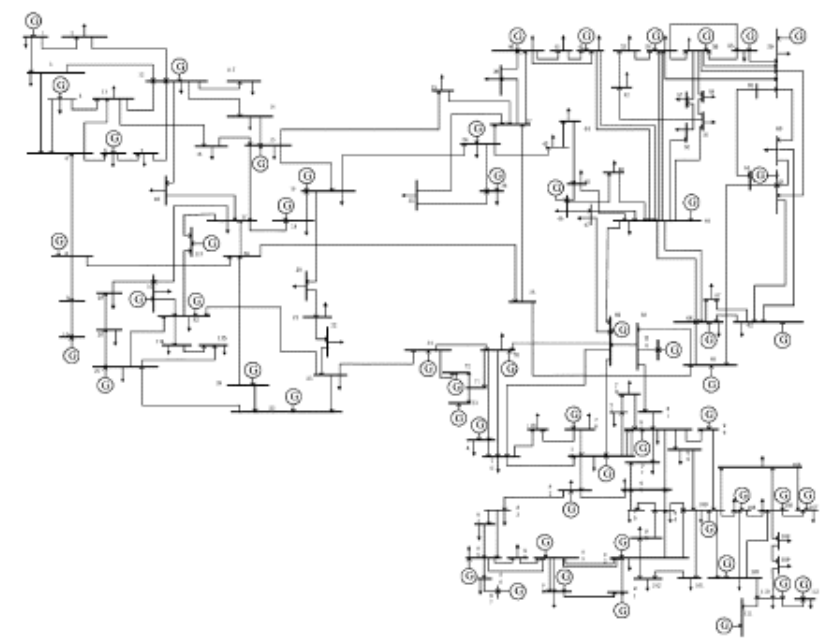

Fig. 9. One-line diagram of IEEE 118-bus system

TABLE II

Scenario 15: HTC with security

\begin{tabular}{l|c}
\hline Unit No & $\begin{array}{c}\text { Daily operating cost: } 769924.1 \$ \\
\text { Hours }(0-24)\end{array}$ \\
\hline $1-3$ & 100000000000000000000000 \\
4 & 11111111111111111111111 \\
5 & 111111111111111111111111 \\
$6-10$ & 1000000000000000000000000 \\
11 & 111111111111111111111111 \\
$12-18$ & 1000000000000000000000000 \\
19 & 11111111111111111110000 \\
$20-21$ & 11111111111111111111111 \\
$22-23$ & 1000000000000000000000000 \\
$24-25$ & 111111111111111111111111 \\
26 & 1000000000000000000000000 \\
$27-29$ & 111111111111111111111111 \\
$30-38$ & 1000000000000000000000000 \\
39 & 1111111100000000000000000 \\
$40-42$ & 1000000000000000000000000 \\
43 & 1110000000000000000000000 \\
$44-45$ & 111111111111111111111111 \\
$46-47$ & 1000000000000000000000000 \\
48 & 1100000000000000000000000 \\
$49-51$ & 1000000000000000000000000 \\
52 & 111111111111111000000000 \\
$53-54$ & 1000000000000000000000000 \\
Hydro1 & 111111111111111111111110 \\
Hydro2 & 111111111111111111111111 \\
Hydro3 & 11111111111111111111100 \\
Hydro4 & 11111111111111111111110 \\
Hydro5 & 111111111111111111111111 \\
Hydro6 & 100000000111111111111100 \\
Hydro7 & 111111111111111111111110 \\
Hydro8 & 111111111111111111111111 \\
\hline &
\end{tabular}


In scenario 15 , the hydro units in all periods of time produce the $14779.110 \mathrm{MW}$ that its average is $615.796 \mathrm{MW}$ at each hour. The minimum generated power at hour 4 is $98.234 \mathrm{MW}$ and the maximum of hydro units generated power at hour 20 is 1262.030 MW. The variation of generated power of hydro plants is very large (1262.030-615.796)/ 615.796 =104.94\%) and generated power by them follows the variations of spot market prices of energy. Also, the amount of whole produced $\mathrm{SO}_{2}$ and $\mathrm{NO}_{\mathrm{x}}$ during 24 hours have been $94224.032 \mathrm{lbs}$ and $47289.168 \mathrm{lbs}$ in the scenario 15 and $103009.373 \mathrm{lbs}$ and $55603.203 \mathrm{lbs}$ in the scenario 8, respectively. This indicates that not only the production cost in the scenario 15 is less than the scenario 8 , but also the amount of gaseous emissions is less. Also, it should be noted that the minimum $\mathrm{SO}_{2}$ and $\mathrm{NO}_{x}$ emissions related to the scenario 6 .

For the purpose of studying the effect of component outages, the proposed adaptive scenario generation scheme, based on the FOR of generating units, is employed to generate scenarios as described in Section II. Forced outage rate of units and branches are taken from [24]. To have better insights on the proposed stochastic framework, in the scenario 15, as the worst case, the online unit with maximum output power; i.e. unit 11 , is considered out of service for 24 hours that leads to increase daily operating cost to 784037.18 \$. Compared with the non-contingent scenario 15 , unit 43 and expensive thermal units 48, 52 have been OFF more hours. Correspondingly, inexpensive thermal unit 10 and unit 19 have been forced to be ON in all of 24 hours and unit 39 is committed at more hours (e.g., 1-10) to compensate for the reduced supply and to satisfy physical constraints.

To thoroughly examine the efficiency of methods, a various range of contingencies including the outage of branches and the trip of generators is chosen in the both single and double forms. The results are shown in tables IV and V. In Tables IV and $\mathrm{V}$, four critical contingencies are applied in scenarios 15 and 8 that have minimum and maximum daily operating cost in case 1 . The single contingency includes single outage of the largest online unit/line during the dispatch period. Since, the spinning and non-spinning reserves provided in the system are more than the output of the largest online generator, the system would be able to withstand the outage of any single unit/line in the system without load shedding but with cost more than no-contingency scenario. Also, in Tables IV-V, the two next contingencies are double contingencies; those scenarios 8 and 15 are with the loss of the largest and second largest online unit and largest online unit and line during the all of 24 hour.

\section{Case2: Stochastic hydrothermal coordination without} considering the security constraints

Again, each 20 scenarios have been simulated without considering the security constraints (inequalities 12,13, and 14) and their power production cost has been compared with each other. It is seen that without considering the security constraints ,the minimum and maximum of daily operating cost are related to the scenario 4 and 11 with $717773.711 \$$ and $838415.736 \$$, respectively. The commitment schedule of the scenario 4 is shown in Table VI. Also, it is obvious that considering the security constraints will cause increasing the
TABLE III ${ }^{*}$

Scenario 8: HTC with security

\begin{tabular}{l|c}
\hline & $\begin{array}{c}\text { Daily operating cost: } 897413 \$ \\
\text { Hours }(0-24)\end{array}$ \\
\hline 1 & 100000000000001111111100 \\
2 & 1111110000000000111111100 \\
3 & 1000000000000000000111100 \\
4 & 10000000011111111111111 \\
5 & 111111111111111111111111 \\
6 & 1100000000000000011111100 \\
7 & 1000000000000000001111100 \\
11 & 11111111111111111111111 \\
12 & 1100000000000000000000000 \\
15 & 1000000000000000000110000 \\
16 & 111111111111111111111100 \\
19 & 1000000000000011111111100 \\
20 & 11111111111111111111111 \\
21 & 100000000111111111111111 \\
24 & 10000000011111111111111 \\
25 & 111111111111111111111111 \\
26 & 1000000000000000000000000 \\
$27-28$ & 11111111111111111111111 \\
29 & 10000000011111111111111 \\
32 & 1110000000000000000000000 \\
37 & 10000000000000000011111 \\
39 & 1111000000000000000000000 \\
40 & 1100000000000000000000000 \\
43 & 1100000000000000000000000 \\
$44-45$ & 11111111111111111111111 \\
53 & 100000000000000000011111 \\
Hydro1 & 111111111111111111111110 \\
Hydro2 & 100000000000111111111111 \\
Hydro3-4 & 111111111111111111111110 \\
Hydro5 & 111100000011111111111111 \\
Hydro6 & 1000000000000000011111110 \\
Hydro7 & 100001111111111111111110 \\
Hydro8 & 1111111111111111111111111 \\
\hline &
\end{tabular}

*: The thermal units, which are not mentioned in the table, had been OFF during all 24 hours

Table IV: Total cost of scenario 15 with single and double contingency (\$)

\begin{tabular}{c|c|c|c|c}
\multirow{2}{*}{ Scenario } & $\begin{array}{c}\text { Single Contingency } \\
\text { Outage of } \\
\text { unit 11 }\end{array}$ & $\begin{array}{c}\text { Outage of } \\
\text { branch 95 }\end{array}$ & $\begin{array}{c}\text { Outage of unit } \\
11 \text { and unit 28 }\end{array}$ & $\begin{array}{c}\text { Outage of unit 11 } \\
\text { and branch 95 }\end{array}$ \\
\hline 15 & $797877.54 \$$ & $788021.36 \$$ & $813484.917 \$$ & $818186.135 \$$ \\
\hline \multicolumn{3}{c}{ Table V: Total cost of scenario 8 with single and double contingency $\$$ \$) } \\
\hline \multirow{2}{*}{ Scenario } & \multicolumn{2}{|c|}{ Single Contingency } & \multicolumn{2}{c}{ Double Contingency } \\
\cline { 2 - 5 } & $\begin{array}{c}\text { Outage of } \\
\text { unit 28 }\end{array}$ & $\begin{array}{c}\text { Outage of } \\
\text { branch 95 }\end{array}$ & $\begin{array}{c}\text { Outage of unit } \\
28 \text { and unit 27 }\end{array}$ & $\begin{array}{c}\text { Outage of unit 28 } \\
\text { and branch 95 }\end{array}$ \\
\hline 8 & $937970.89 \$$ & $886074.50 \$$ & $988481.14 \$$ & $923435.98 \$$ \\
\hline
\end{tabular}

daily power production cost compared to when they are neglected. The main reason is that if some inexpensive units due to satisfying the security constraints cannot be ON, now in this state they can be $\mathrm{ON}$. With comparing the scenario 4 (scenario with lowest cost related to state without considering the security constraints) and the scenario 15 (scenario with lowest cost related to state with considering the security constraints), it is seen that the both inexpensive units 4 and 5 are committed in scenario 15 , while in the scenario 4 only unit 5 maintains its ON state and inexpensive unit 4 only 1 hour of the all of 24 hours has been ON. Also, it is obtained that the expensive thermal unit 52 in scenario 15 to supply the load and satisfy the security constraints has been forced to be $\mathrm{ON}$ at hour 1 to 15 , whereas in scenario 4 this unit all 24 hours has 
not been committed. Compared scenario 4 with scenario 15 , the thermal unit 10 that was OFF in all of 24 hours, has been $\mathrm{ON}$ and unit 39 is committed at the more hours to compensate the reduced supply for decommitting the thermal units 4,19 , 40 and 52 and to satisfy physical constraints in scenario 4 .

With comparing scenario 8 (scenario with highest cost) with and without considering the security constraints, it is seen that the economical unit 5, 45 and inexpensive units 16 and 44 had been forced to be ON over a day to supply the load and satisfy the security constraints, have been OFF. Also expensive thermal units 1, 2, 3 and economical unit 4 and units 19, 37 and 53 that were ON only in the first or last hours of the day, and also without considering the security constraints during these hours they have been OFF and decommitted over a day.

For compensating the reduced supply due to decommiting these units, inexpensive unit 10 and unit 40 that were OFF in all of 24 hours will be $\mathrm{ON}$ at certain hours (e.g., 9-24, 1-24) or unit 3 that was ON only in the 3 first hours of a day, have been forced to be ON over a day to supply the load. Also three units 21,24 and 29 that were OFF in hours 1-8, have been forced to be ON over a day to supply the load.

In this case, when we calculate the HTC solution by excluding transmission and voltage constraints, transmission flow violations in scenario 4 and scenario 11 occur in different lines and hours. Tables VII- VIII show all of transmission flow violations on congested lines for these scenarios without considering security constraints, in which 1 and 0 represent congested/uncongested status of lines at different hours respectively. Table VIII shows that branch 41 is very congested. This branch has $80 \mathrm{MW}$ capacity and is not sufficient to transmit less expensive generation from the righthand side of the system to the left-hand side and its transmission flow in case 2 is near or at the capacity limit at hours 1, 2, 3, 7-11, 14, 15, 19-22. Also Scenario 4 has less congested line than scenario 11, for example congested lines $30,54,127,173$ and 175 in scenario 11 don't have any violated flow in scenario 4 .

TABLE VI: Scenario 4: HTC without security

\begin{tabular}{l|c}
\hline Unit No & $\begin{array}{c}\text { Daily operating cost: } 717773.71 \$ \\
\text { Hours }(0-24)\end{array}$ \\
\hline $1-3$ & 1000000000000000000000000 \\
5 & 1100000000000000000000000 \\
$6-9$ & 111111111111111111111 \\
$10-11$ & 1000000000000000000000000 \\
$12-19$ & 1111111111111111111111111 \\
$20-21$ & 1000000000000000000000000 \\
$22-23$ & 11111111111111111111111 \\
$24-25$ & 1000000000000000000000000 \\
26 & 111111111111111111111 \\
$27-29$ & 1000000000000000000000000 \\
$30-38$ & 11111111111111111111111 \\
$39-40$ & 1000000000000000000000000 \\
$41-43$ & 111111111111111111111111 \\
$44-45$ & 1000000000000000000000000 \\
$46-54$ & 111111111111111111111 \\
Hydro1 & 1000000000000000000000000 \\
Hydro2 & 111111111111111111110 \\
Hydro3 & 11111111111111111111110 \\
Hydro4 & 1111111111111111111110 \\
Hydro5 & 1111111111111111000000 \\
Hydro6 & 10000001111111111111111 \\
Hydro7 & 1100000000111110000000000 \\
Hydro8 & 1111111111111111110000 \\
\hline
\end{tabular}

Case3: Evaluation of effect of hydro units on the SCDHGS problem

Once again, each 20 scenarios have been simulated and investigated. But this time with eliminating the hydro plants problems and then their power production cost that are related to the thermal units, are compared with each other. In this case the minimum and maximum of daily production cost are related to the scenario 15 with $1003371.96 \$$ and scenario 8 with $1193365.26 \$$ respectively. Because of ignoring hydro units which are inexpensive units, it can be seen that daily operating cost in each scenario is higher than two previous cases that are included hydro units. For quick reference, the three case tests are briefly described in Table IX. Also, the aggregated results of the stochastic SCDHGS framework, according to the scenario aggregation procedure, are given in Table X. Using the proposed stochastic framework; all 20 accepted scenarios contribute into determining the SCDHGS results according to their probability values. Finally, the number of variables and constraints and solution time for the three cases are presented in table XI. From the Table XI, it takes 35540 seconds to find the optimal solutions of the case lof the problem. This is mainly for dimensionality issue which includes millions of equations, continuous and discrete variables.

TABLE VII

Congested/uncongested status of violated lines in scenario 4 (with minimum cost) of case 2

Hours $(0-24)$

L37 0000000101100110001010000

L41 0000000101100110001010000

L115 0000000000000010000000000

L123 0100000101111110001010000

L124 0100000101000010001010000

L126 0100000101000000001010000

L134 0100000101000010001010000

L136 0100000101000010001010000

L137 0100000101000010001010000

L138 0100000101000000001010000

L139 0100000100000000001010000

L142 0000000000111110000000000

TABLE VIII

Congested/uncongested status of violated lines in scenario 11 (with maximum cost) of case 2

Hours (0-24)

L30 0000000010100000000000000

L37 0000001110101111000000000

L41 0111000111110011000111100

L54 0000001010100000000000000

L123 0000001010100000010111100

L124 0000001110100111000000000

L126 0000001010100110000000000

L127 0000000000100000000000000

L134 0000001110100111000000000

L136 0000001110100111000000000

L137 0000000010100000000000000

L138 0000000010100000000000000

L139 0000000010100000000000000

L142 0000000000000000010111100

L173 0000000000000100010000000 
Table IX : Scenarios with minimum/maximum total cost $(\$)$ in 3 cases

\begin{tabular}{c|c|c|c|c|c|c}
\hline \multirow{2}{*}{ Scenario } & \multicolumn{2}{|c|}{ With security constraints } & \multicolumn{2}{c}{ Without security constraints } \\
\cline { 2 - 7 } & Case 1 & Case 2 & Case 3 & Case 1 & Case 2 & Case 3 \\
\hline 15 & 769924 & --- & 1003371 & ----- & -------- & ------ \\
\hline 8 & 897413 & --- & 1193365 & ---- & 787426 & ------ \\
\hline 4 & -------- & ------ & --------- & ----- & 717773 & ----- \\
\hline 11 & -------- & --- & 1171508 & ----- & 838415 & ----- \\
\hline
\end{tabular}

Table X: Aggregated results of the stochastic SCDHGS framework in 3 cases

\begin{tabular}{c|c|c|c}
\hline & Case 1 & Case 2 & Case 3 \\
\hline Daily operating cost $(\$)$ & 810273.845 & 752056.419 & 1099294.396 \\
\hline
\end{tabular}

Table XI: Optimization statistics for all three cases

\begin{tabular}{c|c|c|c|c}
\hline Case & Variables & Discrete Variables & Equations & Solution time $(\mathrm{Sec})$ \\
\hline Case 1 & 1380080 & 602040 & 1778820 & 35540 \\
\hline Case 2 & 1322480 & 594250 & 1544420 & 1840 \\
\hline Case 3 & 1290560 & 567480 & 1594180 & 32620 \\
\hline
\end{tabular}

Also from this table, one can see that the execution time of the problem is dramatically decreased as the security constraints are inactivated in the case 2. This matter shows that some constraints are critical and can be relaxed using decomposition techniques. In addition to decomposition techniques, the parallel computation approach can significantly decrease this solution time. However, this paper pertains to present the comprehensive model for the stochastic security constrained DHGS problem rather than computational viewpoints of the problem.

\section{Conclusions}

This paper presents a stochastic security constrained DHGS framework in the form of MIP optimization problem in which the valve loading effect cost, dynamic ramp rate, POZs, fuel limitation are modeled all in linear form. It also includes multi-performance curves with spillage and time delay between reservoirs for hydro units making the DHGS framework more realistic. The stochastic nature of proposed security constrained DHGS structure considers generating units and branches contingencies and load uncertainty. Furthermore, security aspects of the power system as one of main responsibilities of the ISOs are incorporated in the stochastic security constrained DHGS as extra objective functions. With the proposed method, ISOs can cope with the uncertainties of the DHGS problem, i.e. load forecast error and branches and generating units' outages. Besides, the proposed method can consider the security constrained DHGS problem in such a way that the ISO's concerns about the system security are relieved with tolerable and reasonable total cost. The other main feature of the proposed framework refers to the linear nature of the formulations which is very important for application of the model in the large scale and the real size power system. Therefore, the proposed scheme is practical to generate appropriate information for ISOs to decides how much power generate by each generator. The main disadvantage of the proposed framework is the computational burden of the problem solution which can be solved using parallel computation, efficient scenario reduction techniques and decomposition methods. Accordingly, the research work under way to a) present a stochastic model with other scenario reduction techniques and b) use accelerated benders decomposition to reduce computational burden.

\section{References}

[1] A. Wood and B. Wollenberg, "Power Generation, Operation, and Control" .New York: Wiley, 1996.

[2]X. Guan, E. Ni, R. Li, P.B. Luh, “An optimization-based algorithm for scheduling hydrothermal power systems with cascaded reservoirs and discrete hydro constraints, " IEEE Trans. Power Syst., vol. 12, no. 4, pp. 1775-1780, 1997.

[3]J.-S. Yang, N. Chen, "Short term hydrothermal coordination using multipass dynamic programming," IEEE Trans. Power Syst., vol. 4, no. 3, pp. 1050-1056, 1989.

[4]M. Giuntoli, D. Poli, "A novel mixed-integer linear algorithm to generate unit commitment and dispatching scenarios for reliability test grids," International Review Electrical Engineering (IREE), vol. 6. no. 4, pp. 1971-1983, June 2011

[5]W.S. Sifuentes, A. Vargas, "Hydrothermal scheduling using benders decomposition: accelerating techniques," IEEE Trans. Power Syst., vol. 22, no. 3, pp. 1351-1359, 2007

[6]C.Srinivasa Rao et al, "A modified genetic approach to hydrothermal system with thyristor controlled phase shifter under open market system" International Review Electrical Engineering (IREE), vol. 2, no. 1, pp. 507-514, August 2007.

[7]M. Gavrilas, V. Stahie, "Cascade hydropower plants optimization with honey bee mating optimization algorithm," International Review Electrical Engineering (IREE), vol. 6, no. 5, pp, 24-56, October 2011.

[8]C.E. Zoumas, A.G. Bakirtzis, J.B. Theocharis, V. Petridis, "A genetic algorithm solution approach to the hydrothermal coordination problem," IEEE Trans Power Syst., vol. 19, no. 3, pp. 1356-1364, 2004.

[9]I. A. Farhat and M. E. El-Hawary, "Optimization methods applied for solving the short-term hydrothermal coordination problem," Elect. Power Syst. Res., vol. 79, no. 9, pp. 1308-1320, Sep. 2009.

[10] M. Shahidehpour, H. Yamin, and Z. Y. Li, "Market operations in electric power system”. New York: Wiley, 2002

[11] Y. Fu, M. Shahidehpour, and Z. Li, "AC contingency dispatch based on security- constrained unit commitment," IEEE Trans. Power Systems, vol. 21, no. 2, pp. 897 -908, May 2006.

[12] J. M. Arroyo, and A. J. Conejo, "Optimal response of a thermal unit to an electricity spot market," IEEE Trans. Power Syst., vol. 15, no. 3, pp. 1098-1104, Aug. 2000.

[13] A.J. Conejo, J.C. Contreras, J.M. Arroyo, S. de la Torre, "Optimal response of an oligopolistic generating company to a competitive poolbased electric power market”, IEEE Trans. Power Syst., vol. 17, no. 2, pp. 424-430, 2002.

[14] N. Amjady, H. R. Soleymanpour, "Daily hydrothermal generation scheduling by a new modified adaptive particle swarm optimization technique", Electric Power Systems Research, vol. 80, no. 6, pp. 723732, June 2010,

[15] G.Dany, "Kraftwerksreserve in elektrischen verbundsystemen mit hohem windenergieanteil (in german)," Ph.D. dissertation, Germany, 2000.

[16] B.C. Ummels, M. Gibescu, E. Pelgrum, W.L. Kling, A.J. Brand, "Impacts of wind power on thermal generation unit commitment and dispatch,” IEEE Trans Energy Conversion, vol. 22, no. 1, pp. 44-51, March 2007.

[17] A.J. Conejo, J.M, J. Contreras, F.A. Villamor, "Self-scheduling of a hydro producer in a pool-based electricity market," IEEE Trans. Power Syst., vol. 17, no. 4, pp. 1265-1272, 2002. 
[18] J.G. Gonzalez, G.A. Castro, "Short-term scheduling with cascaded and head-dependent reservoirs based on mixed-integer linear programming," IEEE Power Tech '01 Conference, Porto, Portugal, September, 2001.

[19] K. Meng, H. G. Wang, Z. Y. Dong, and K. P. Wong, "Quantum-inspired particle swarm optimization for valve-point economic load dispatch," IEEE Trans. Power Syst., vol. 25, no. 1, pp. 215-222, Feb. 2010.

[20] N. Amjady and H. Nasiri-Rad, "Nonconvex economic dispatch with AC constraints by a new real coded genetic algorithm," IEEE Trans. Power Syst., vol. 24, no. 3, pp. 1489-1502, Aug. 2009.

[21] M. R. AlRashidi and M. E. El-Hawary, "Hybrid particle swarm optimization approach for solving the discrete OPF problem considering the valve loading," IEEE Trans. Power Syst., vol. 22, no. 4, pp. 2030 2038, Nov. 2007.

[22] T. Li, and M. Shahidehpour, "Dynamic ramping in unit commitment," IEEE Trans. Power Syst., vol. 22, no. 3, pp. 1379-1381, Aug 2007.

[23] B. Dursun, B. Alboyaci, C. Gokcol, "Optimal wind-hydro solution for the Marmara region of Turkey to meet electricity demand," Energy, vol. 36 , no. 2, pp. 864-872, 2011 .

[24] Lei Wu, Mohammad Shahidehpour, and Tao Li, "Stochastic securityconstrained unit commitment, " IEEE Trans. Power Syst., vol. 22, no. 2 pp. 800-811, May 2007.

[25] F. D. Galiana, F. Bouffard, J. M. Arroyo, and J. F. Restrepo, "Scheduling and pricing of coupled energy and primary, secondary, and tertiary reserves," Proc. IEEE, vol. 93, no. 11, pp. 1970-1983, Nov. 2005.

[26] K. W. Cheung, P. Shamsollahi, D. Sun, J. Milligan, and M. Potishnak, "Energy and ancillary service dispatch for the interim ISO New England electricity market,” IEEE Trans. Power Syst., vol. 15, no. 3, pp. 968 974, Aug. 2000.

[27] Y. Liu, Z. Alaywan, M. Rothleder, S. Liu, and M. Assadiam, "A rational buyer's algorithm used for ancillary service procurement," in Proc. IEEE PES Summer Meeting, Singapore, 2000.

[28] R. Billinton and R. N. Allan, "Reliability evaluation of power systems," 2nd ed. New York: Plenum, 1996.

[29] L. Wu, M. Shahidehpour, and T. Li, "Cost of reliability analysis based on stochastic unit commitment," IEEE Trans. Power Syst., vol. 23, no. 3, pp. 1364-1374, Aug. 2008.

[30] Z. Michalewicz, "Genetic algorithm + data structure = evalution program,” New York: Springer-Verlag, 1996.

[31] I. G. Damousis, A. G. Bakirtzis, and P. S. Dokopolous, "A solution to the unit-commitment problem using integer coded genetic algorithm," IEEE Trans. Power Syst., vol. 19, no. 1, pp. 198-205, Feb. 2003.

[32] J. Aghaei, H.A. Shayanfar , N. Amjady, "Joint market clearing in a stochastic framework considering power system security," Applied Energy, vol. 86, no. 9, pp. 1675-1682, 2009.

[33] T. Li, M. Shahidehpour, and Z. Li, "Risk-constrained bidding strategy with stochastic unit commitment," IEEE Trans. Power Syst., vol. 22, no. 1, pp. 449-458, Feb. 2007

[34] N. Amjady, J. Aghaei, H.A. Shayanfar, "Stochastic multiobjective market clearing of joint energy and reserves auctions ensuring power system security," IEEE Trans Power Syst., vol. 24, no. 4, pp. 18411854, Nov. 2009.

[35] Y. Fu, "Security-constrained operational planning in power systems", $\mathrm{PhD}$ thesis, Illinois Institute and Technology, May 2006.

[36] M. Parvania, M. Fotuhi-Firuzabad, "Demand response scheduling by stochastic SCUC," IEEE Trans. Smart Grid., vol. 1, no. 1, pp. 89-98, June. 2010.

[37] S. Bisanovic, M. Hajro, and M. Dlakic, "Hydrothermal self-scheduling problem in a day-ahead electricity market," Elect. Power Syst. Res., vol. 78, no. 9, pp. 1579-1596, Sep. 2008.

[38] T. Li, and M. Shahidehpour, "Price-based unit commitment: a case of lagrangian relaxation versus mixed integer programming," IEEE Trans. Power Syst., vol. 20, no. 4, pp. 2015-2025, Nov. 2005.

[39] J.B. Park, Ki-S. Lee, J-R. Shin, K.Y. Lee, "A particle swarm optimization for economic dispatch with non-smooth cost functions", IEEE Trans. Power Syst., vol. 20, no. 1, pp. 34-42, Feb 2005.
[40] M. W. Li, Y. P. Li, G. H. Huang, "An interval-fuzzy two-stage stochastic programming model for planning carbon dioxide trading under uncertainty," Energy, vol. 36, no. 9, pp. 5677-5689, 2011.

[41]W. Lei, M. Shahidehpour, and L. Tao, "GENCO's risk-based maintenance outage scheduling," IEEE Trans. Power Syst., vol. 23, no. 1, pp. 127-136, Feb. 2008.

[42] J. Aghaei, N. Amjady and H. A. Shayanfar, "Demand-side reserve in stochastic market clearing of joint energy/reserve auctions," Euro Trans Electr Power, vol. 21, no. 1, pp. 565-580, 2010

[43] [Online]. Available: http://motor.ece.iit.edu/data/SCUC_118test.xls

[44] V. Vahidinasab, S. Jadid, "Stochastic multiobjective self-scheduling of a power producer in joint energy and reserves markets," Electric Power Systems Research, vol. 80, no.7, pp. 760-769, 2010.

[45] T. Li, and M. Shahidehpour, "Risk-constrained generation asset arbitrage in power systems," IEEE Trans. Power Syst., vol. 22, no. 3, pp. 1330-1339, Aug. 2007

[46] Z. Li, and M. Shahidehpour, "Security-constrained unit commitment for simultaneous clearing of energy and ancillary services markets," IEEE Trans. Power Syst., vol. 20, no. 2, pp. 1079-1088, May. 2005.

[47] Generalized Algebraic Modeling Systems (GAMS), [Online] Available: http://www.gams.com

[48] F. Bouffard, F. Galiana, and A. Conejo, "Market-clearing with stochastic security-Part I: Formulation,” IEEE Trans. Power Syst., vol. 20, no. 4, pp. 1818-1826, Nov. 2005.

[49] F. Bouffard, F. Galiana, and A. Conejo, "Market-clearing with stochastic security_Part II: Case studies,” IEEE Trans. Power Syst., vol. 20, no. 4, pp. 1827-1835, Nov. 2005 